\title{
PREFACE: 11TH WORKSHOP ON NON-CLASSICAL MODELS OF AUTOMATA AND APPLICATIONS (NCMA 2019)
}

\author{
Markus Holzer ${ }^{1}$ And José M. Sempere ${ }^{2, *}$
}

Accepted July 5, 2021.

Many non-classical automata models are natural objects of theoretical computer science. They are studied from different points of view in various areas, both as theoretical concepts and as formal models for applications. The aim of the NCMA workshop series is to bring together researchers working on different aspects of different variants of non-classical automata models in order to exchange and develop novel ideas. A deeper and interdisciplinary coverage of this particular area may gain new insights and substantial progress.

This special issue of RAIRO-ITA contains six contributions reported at the 11th International Workshop on Non-Classical Models of Automata and Applications (NCMA 2019) that took place in Valencia (Spain) on 2nd and 3rd July 2019, organized by the Research Group on Formal Automata and their Applications of the VRAIN institute of the Universitat Politècnica de València.

The first workshop, NCMA 2009, was held in Wroclaw, Poland, in 2009 as a satellite event of FCT 2009, sponsored by the AutoMathA project of the European Science Foundation (ESF). The second workshop, NCMA 2010, was held in Jena, Germany, as an associated workshop of the Conference on Membrane Computing (CMC 2010). Since 2011, the NCMA workshops have been organized as stand-alone events, NCMA 2011 in Milan, Italy, NCMA 2012 in Fribourg, Switzerland, NCMA 2013 in Umeå, Sweden, NCMA 2014 in Kassel, NCMA 2015 in Porto, Portugal, NCMA 2016 in Debrecen, Hungary, NCMA 2017 in Prague, Czech Republic, and NCMA 2018 in Košice, Slovakia.

During the NCMA 2019, 11 full contributions and three short contributions were accepted and presented. In addition, there were two invited talks given by Prof. Dr. Victor Mitrana (Univ. Politécnica de Madrid) and Dr. Agustín Riscos-Núñez (Univ. de Sevilla). The works presented at the workshop were selected and reviewed by the members of the Program Committee. We thank the members of the Program Committee for their excellent work: José M. Sempere (Universitat Politècnica de València, Valencia, Spain) Markus Holzer (University of Giessen, Germany), Erzsébet Csuhaj-Varjú (Eötvös Loránd University, Budapest, Hungary), Henning Fernau (Trier University, Germany), Bruno Guillon (INRIA Lille, France), Galina Jirásková (Slovak Academy of Sciences, Slovakia), Lila Kari (University of Waterloo, Canada), Zbyněk Křivka (Brno University of Technology, Czech Republic), Alberto Leporati (Università degli Studi di Milano - Bicocca, Italy), Andreas Malcher (University of Giessen, Germany), Brink van der Merve (University of Stellenbosch, South Africa), Benedek Nagy (Eastern Mediterranean University, Famagusta, North Cyprus), Friedrich Otto (University of Kassel, Germany), Cem Say (Boğazici University of Istanbul, Turkey), Petr Sosík (Silesian University, Opava, Czech Republic), Bianca

1 Justus-Liebig-Universität Gießen, Germany.

2 Universitat Politècnica de València, Spain.

* Corresponding author: jsempere@dsic.upv.es

(C) The authors. Published by EDP Sciences, 2021 
Truthe (University of Giessen, Germany) and György Vaszil (University of Debrecen, Hungary). In addition, we are grateful to the members of the Organizing Committee from the Universitat Politécnica de Valéncia: Damián López, Marcelino Campos, Antonio Larriba and Manuel Vázquez de Parga.

From all the contributions submitted, a small number of them were selected according to the subject matter and quality of the results presented in them. The authors of these contributions were invited to review again all the material presented at NCMA 2019 and to extend their work significantly. As a result, and after a meticulous review of the submitted papers, we present in this issue of the RAIRO-ITA journal the six selected, revised and extended works.

In the special issue, we have the following works: "Synchronizing Series-Parallel Deterministic Finite Automata with Loops and Related Problems" by Jens Bruchertseifer and Henning Fernau related to Deterministic Finite Automata and synchronizing words, "On Deterministic 1-Limited 5' -> 3' Sensing Watson-Crick finite state transducers" by Benedek Nagy and Kovács Zita related to some WKFA models and transducers, "Accepting Networks of Evolutionary Processors with Resources Restricted and Structure Limited Filters" by Jurgen Dassow and Bianca Truthe dealing with some restrictions on filters in Networks of Evolutionary Processors, "Bottom-Up Derivatives and Derived Terms from Tree Expressions" by Samira Attou, Ludovic Mignot and Djelloul Ziadi which extends the notions of derivatives to the case of tree derivatives, "Digging Input-Driven Pushdown Automata" by Martin Kutrib and Andreas Malcher on pushdown automata with special actions on the pushdown store and, finally, "On restarting automata with auxiliary symbols and small window size" by Frantisek Mraz and Friedrich Otto on the relationship between window size and restarting automata. All selected papers reflect very well the spirit of the NCMA workshop series.

We would like to thank first of all the authors of the papers as well as the reviewers of the papers presented in this issue. The revisions have been carried out during a particularly difficult year under the circumstances caused by the global COVID-19 epidemiological crisis which has affected the whole process of revising, proofreading and editing across the board. We would also like to show our gratitude to the RAIRO-ITA staff who, for the same reasons, have shown great understanding and professionalism in order for us to conclude this whole process in the best possible way.

We thank them all. 